\title{
Influence of Guest/Host Morphology on Room Temperature Phosphorescence Properties of Pure Organic Doped Systems
}

Xiaoqing Liu, ${ }^{\text {a }}$ Yanyan Pan, ${ }^{\text {a }}$ Yunxiang Lei, ${ }^{* a}$ Nannan Liu, ${ }^{\text {a }}$ Wenbo Dai, ${ }^{\text {b }}$ Miaochang Liu, ${ }^{\text {a }}$ Zhengxu Cai, ${ }^{* b}$ Huayue Wu, ${ }^{a}$ Xiaobo Huang, ${ }^{* a}$ and Yuping Dong ${ }^{b}$

a School of Chemistry and Materials Engineering, Wenzhou University. Wenzhou 325035 (P. R. China)

${ }^{\mathrm{b}}$ School of Materials Science \& Engineering, Beijing Institute of Technology, Beijing, 10081 (P. R. China)

E-mail: yunxianglei@wzu.edu.cn (Y. Lei); caizx@bit.edu.cn (Z. Cai); xiaobhuang@wzu.edu.cn (X. Huang).

\section{Materials and characterization}

${ }^{1} \mathrm{H}$ NMR and ${ }^{13} \mathrm{C}$ NMR spectra were carried out by a Bruker ARX500 spectrometer with $\mathrm{CDCl}_{3}$ and DMSO- $d_{6}$ as the solvent. UV-vis absorption spectra were measured by a Persee TU-1901 spectroscopy. Fluorescence spectra were measured by a Hitachi F7000 spectrophotometer. Phosphorescence spectra were measured by a FLS920 lifetime and steady state spectrometer. X-Ray crystal structure analyses were conducted on a Bruker-AXS SMART APEX2 CCD diffractometer. Solid-state emission quantum yields (QY) were collected on a FluoroMax-4 (Horiba Jobin Yvon) fluorimeter equipped with integrated sphere. The theoretical ground-state geometry and electronic structure were performed using the density functional theory (DFT) with B3LYP hybrid functional at the basis set level of $6-31+\mathrm{G}(\mathrm{d}, \mathrm{p}) .{ }^{1}$ All the theoretical calculations were optimized using Gaussian 09 package. Phenyl(pyridin-2-yl)methanone, phenyl (pyridin-3-yl)methanone, and di(yridin-2-yl)methanone were purchased from commercial suppliers. 8-Hydroxy-3,6-dimethyl-1-(pyrrolidin-1-yl)isoquinoline-7carbonitrile (3a), 8-hydroxy-3,6-dimethyl-1-(piperidin-1-yl)isoquinoline-7carbonitrile (3b), and 1-(azepan-1-yl)-8-hydroxy-3,6-dimethylisoquinoline-7carbonitrile (3c) were synthesized using 2,6-dimethyl-4H-pyran-4-one (1) as the starting materials according to the previous literature. ${ }^{2}$

\section{References:}

1. M. J. Frisch, G. W. Trucks, H. B. Schlegel, G. E. Scuseria, M. A. Robb, J. R. Cheeseman, J. A.Montgomery, T. Vreven, K. N. Kudin, J. C. Burant, et al. Gaussian 03, revision E.01; Gaussian, Inc.: Wallingford, CT, 2004.

2. (a) Y. Chen, X. Zhang, M. Wang, Y. Zhou, M. Liu, X. Huang, H. Wu, J. Mater. Chem. C 2019, 7, 12580-12587; (b) X. Zhang, Y. Zhou, M. Wang, Y. Chen, Y. Zhou, 
W. Gao, M. Liu, X. Huang, H. Wu, Chem. Asian J. 2020, 15, 1692-1700.

\section{Synthesis of target compounds}
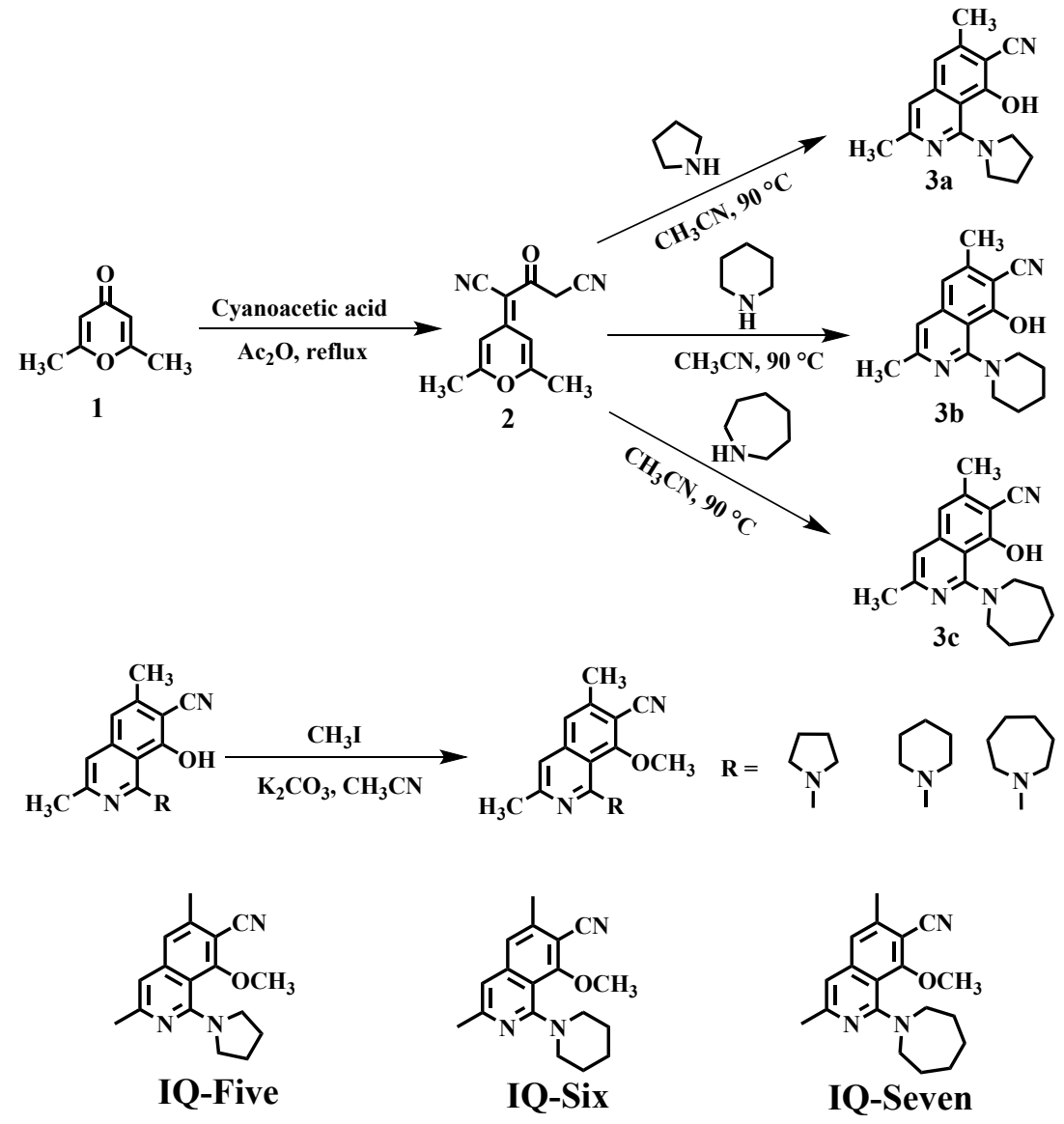

Scheme S1. Synthetic routes of three guest componuds.

\section{Synthesis of the guest molecules}

General procedure: A mixture of $\mathbf{3 a} / \mathbf{3 b} / \mathbf{3 c}(10.0 \mathrm{mmol})$, iodomethane $(30.0 \mathrm{mmol})$, and $\mathrm{K}_{2} \mathrm{CO}_{3}(2.0 \mathrm{mmol})$ in $10.0 \mathrm{~mL}$ acetonitrile was heated at $80^{\circ} \mathrm{C}$ for $5 \mathrm{~h}$. The solvent was removed under reduced pressure, and then the residue was purified by column chromatography (petroleum ether/ethyl acetate $=1: 10, \mathrm{v}: \mathrm{v}$ ) to afford the pure target compounds.

8-Methoxy-3,6-dimethyl-1-(pyrrolidin-1-yl)isoquinoline-7-carbonitrile (IQ-Five): White solid. Yield: $84 \%$, m. p. $124.9^{\circ} \mathrm{C}-125.4^{\circ} \mathrm{C} .{ }^{1} \mathrm{H}$ NMR (DMSO- $d_{6}, 500 \mathrm{MHz}$ ): $\delta$ $7.28(\mathrm{~s}, 1 \mathrm{H}), 6.79(\mathrm{~s}, 1 \mathrm{H}), 3.76(\mathrm{~s}, 3 \mathrm{H}), 3.51(\mathrm{~s}, 4 \mathrm{H}), 2.46(\mathrm{~s}, 3 \mathrm{H}), 2.35(\mathrm{~s}, 3 \mathrm{H}), 1.82(\mathrm{~s}$, 4H) ppm. ${ }^{13} \mathrm{C} \mathrm{NMR}\left(\mathrm{CDCl}_{3}, 125 \mathrm{MHz}\right): \delta 162.9,156.1,153.2,143.9,140.4,120.9$, $116.4,108.6,108.3,102.0,62.2,50.7,25.4,24.1,20.5 \mathrm{ppm}$.

8-Methoxy-3,6-dimethyl-1-(piperidin-1-yl)isoquinoline-7-carbonitrile (IQ-Six): White solid. Yield: $86 \%$, m. p. $146.2^{\circ} \mathrm{C}-146.7^{\circ} \mathrm{C} .{ }^{1} \mathrm{H} \mathrm{NMR}\left(\mathrm{CDCl}_{3}, 500 \mathrm{MHz}\right): \delta 7.19$ (s, 1H), 6.82 (s, 1H), 3.93 (s, 3H), 3.56 (br, 2H), 3.02 (br, 2H), 2.56 (s, 3H), 2.48 (s, 
3H), 1.76-1.62 (m, 6H) ppm. $\left.{ }^{13} \mathrm{C} \mathrm{NMR} \mathrm{(} \mathrm{CDCl}_{3}, 125 \mathrm{MHz}\right): \delta 162.8,159.1,153.0,143.9$, $140.5,122.5,116.3,111.5,110.7,104.5,63.6,52.6,26.1,24.7,24.2,20.6$ ppm.

1-(Azepan-1-yl)-8-methoxy-3,6-dimethylisoquinoline-7-carbonitrile (IQ-Seven):

White solid. Yield: $82 \%$, m. p. 126.6-127. $3^{\circ} \mathrm{C} .{ }^{1} \mathrm{H}$ NMR (DMSO- $d_{6}, 500 \mathrm{MHz}$ ): $\delta 7.32$ $(\mathrm{s}, 1 \mathrm{H}), 6.82(\mathrm{~s}, 1 \mathrm{H}), 3.76(\mathrm{~s}, 3 \mathrm{H}), 3.60(\mathrm{t}, 4 \mathrm{H}), 2.46(\mathrm{~s}, 3 \mathrm{H}), 2.36(\mathrm{~s}, 3 \mathrm{H}), 1.71(\mathrm{~s}, 4 \mathrm{H})$, $1.51(\mathrm{~s}, 4 \mathrm{H}) \mathrm{ppm} .{ }^{13} \mathrm{C} \mathrm{NMR}\left(\mathrm{CDCl}_{3}, 125 \mathrm{MHz}\right): \delta 162.7,157.9,152.5,144.3,140.2$, 121.3, 116.4, 109.1, 108.1, 102.4, 62.5, 52.6, 28.3, 27.8, 24.1, 20.5 ppm.

\section{Preparation methods of doped materials}

Put the corresponding amount of host and guest together and heat it to $60{ }^{\circ} \mathrm{C}$ in air atmosphere. After the guests are completely dissolved in the molten hosts, the mixed systems are cooled to room temperature, and the mixed systems are crystallized to obtain the doped materials. The doped materials with high guest-host molar ratio (1:10, $1: 100,1: 100)$ are using direct weighing method, while for guest-host molar ratio (1:10000) doped materials, we use the indirect dilution method.

\section{Figures and tables}

(a)

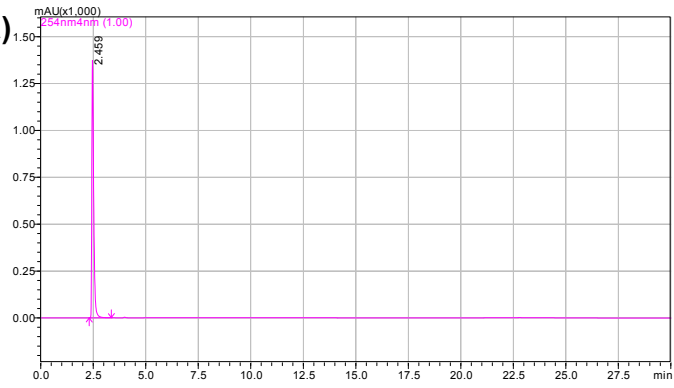

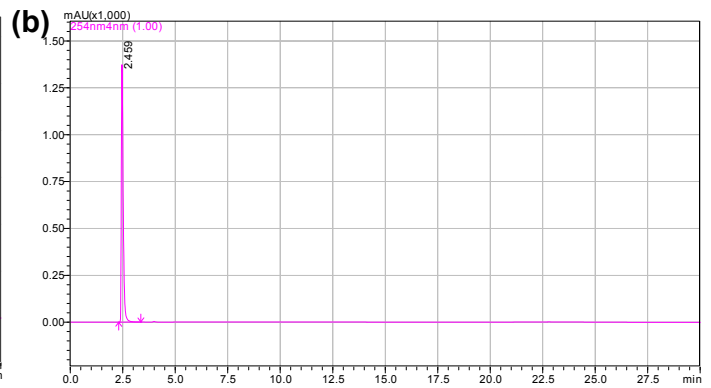

(c)

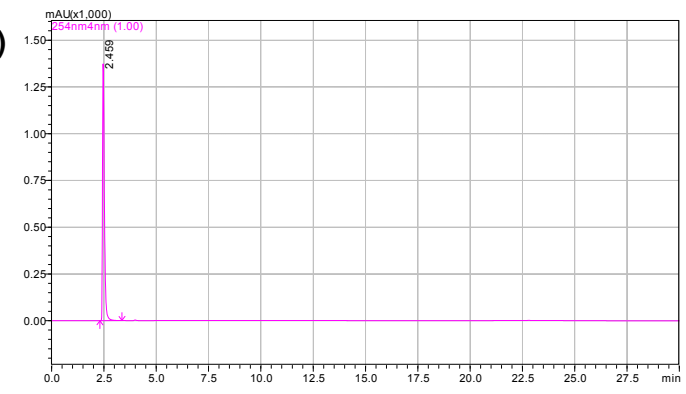

Figure S1. High performance liquid chromatography of IQ-Five (a), IQ-Six (b), IQSeven (c) $\left(\mathrm{CH}_{3} \mathrm{OH} / \mathrm{H}_{2} \mathrm{O}=80 \%: 20 \%\right)$. 


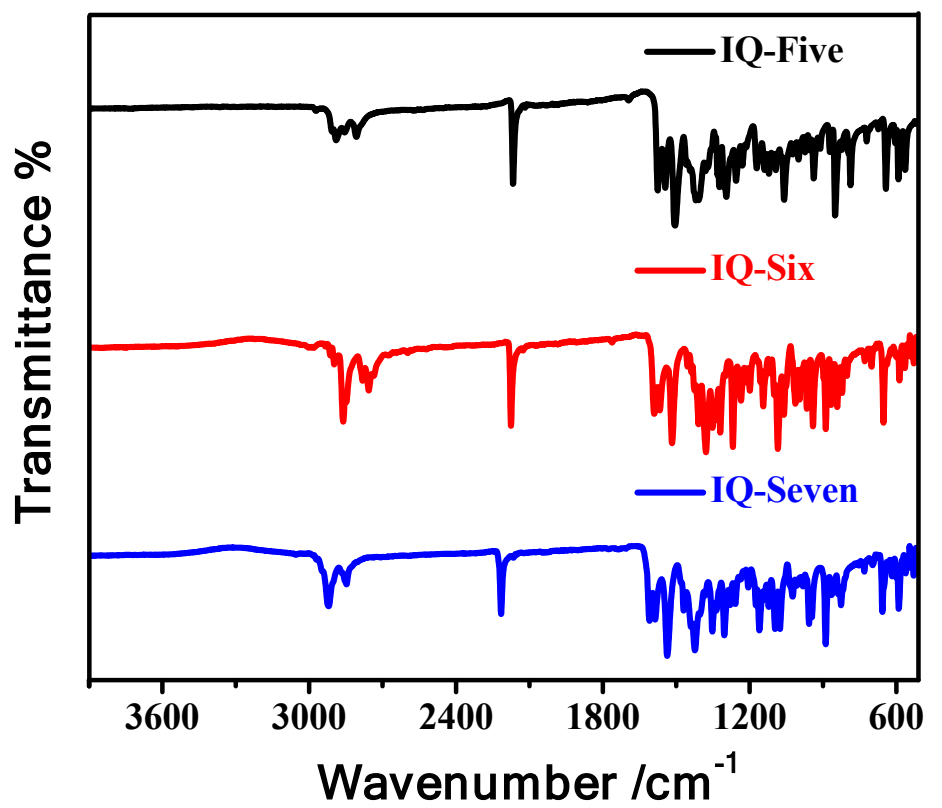

Figure S2. Infrared absorption of three guests.
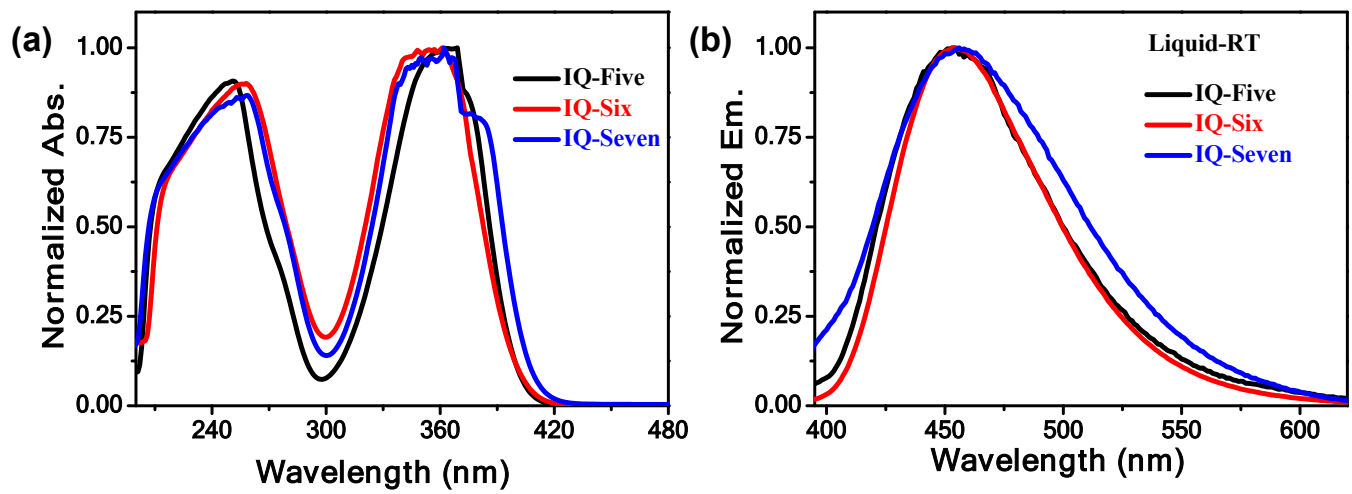

Figure S3. Normalized absorption (a) and emission (b) spectra of the guests in THF solvent. Concentration: $1.0 \times 10^{-5} \mathrm{~mol} / \mathrm{L}$.

Table S1. Emission quantum yields of the hosts.

\begin{tabular}{|l|c|c|c|}
\hline & 2PPO & 3PPO & DPyO \\
\hline Fluo. QYs (\%) & 1.3 & 3.4 & 1.5 \\
\hline Phos. QYs (\%) & $<0.1$ & $<0.1$ & $<0.1$ \\
\hline
\end{tabular}



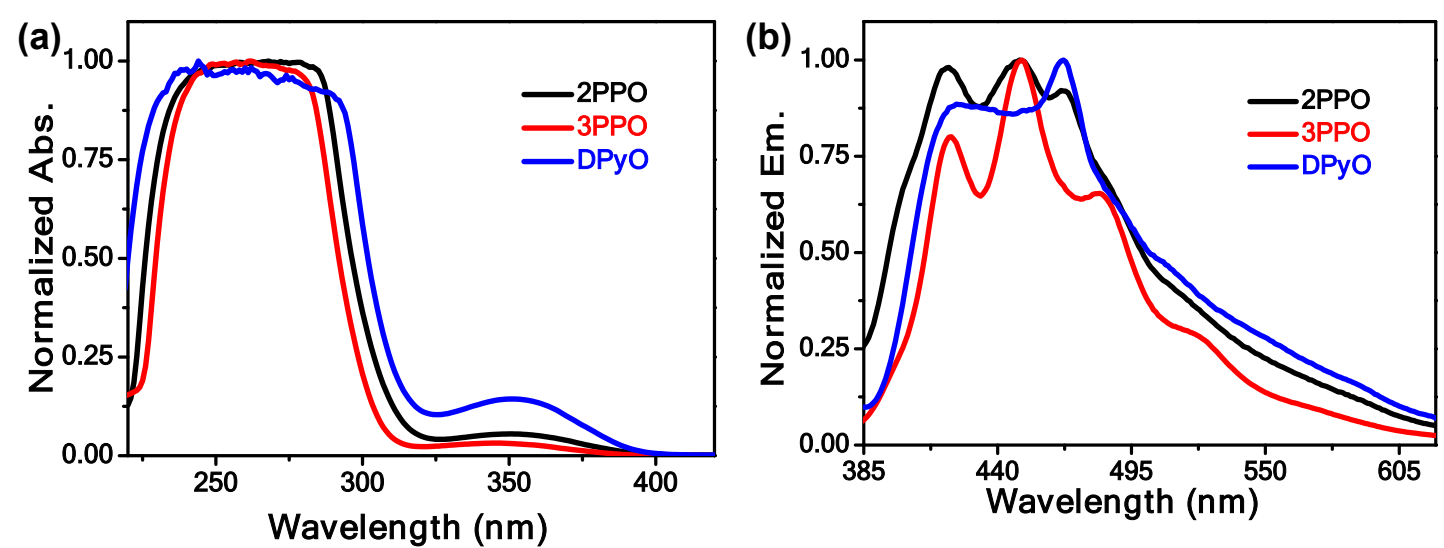

Figure S4. Normalized absorption (a) and emission (b) spectra of the hosts in THF solvent. Concentration: $1.0 \times 10^{-5} \mathrm{~mol} / \mathrm{L}$.

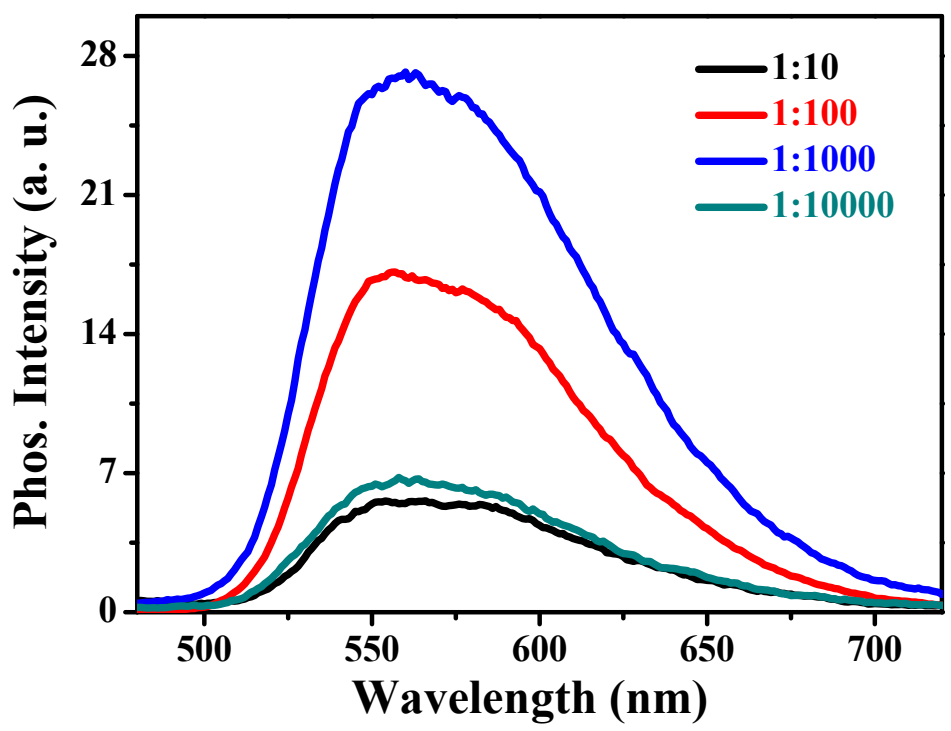

Figure S5. Delayed emission spectra of IQ-Five/2PPO doped materials with different amounts of IQ-Five.

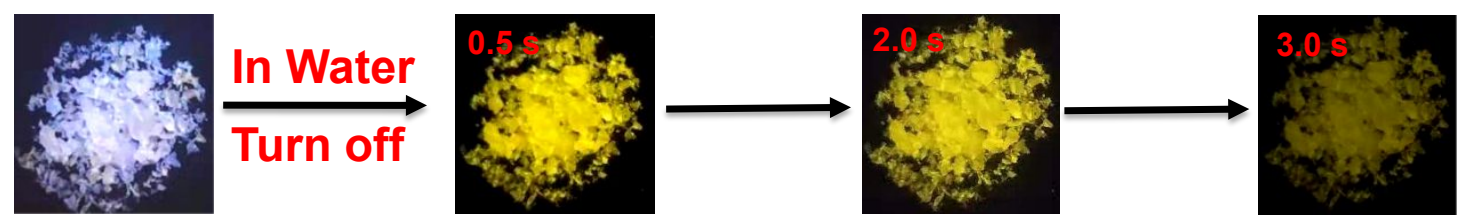

Figure S6: Phosphorescence photo of IQ-Six/2PPO in water. 

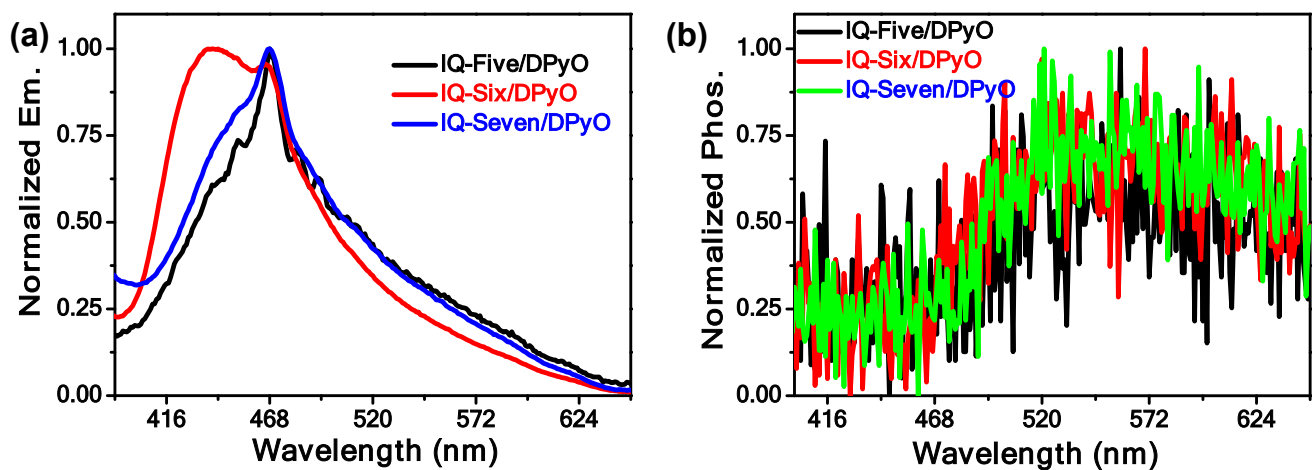

Figure S7. Fluorescence (a) and phosphorescence (b) spectra of IQ-Five/DPyO, IQSix/DPyO, and IQ-Seven/ DPyO.

Table S2. Average particle size and polydispersity index (PDI) of IQ-Six in THF-water mixtures with different $f_{w}$.

\begin{tabular}{lccc}
\hline Compound & $f_{\mathrm{w}}$ & Average particle size $(\mathrm{nm})$ & PDI \\
\hline IQ-Six & $0 \%$ & 14.2 & 0.204 \\
& $30 \%$ & 207.0 & 0.538 \\
& $60 \%$ & 206.5 & 0.636 \\
& $90 \%$ & 205.2 & 0.427 \\
& $99 \%$ & 4746 & 0.724 \\
\hline
\end{tabular}

(a)

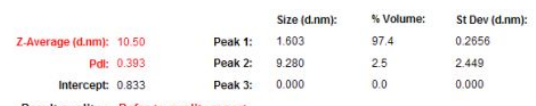

(b)

$\begin{array}{rllll} & & \text { Size (d.nm): } & \text { \% Volume: } & \text { St Dev (d.nm } \\ \text { Z-Average (d.nm): } 106.3 & \text { Peak 1: } & 874.6 & 0.2 & 230.3 \\ \text { Pdl: } 1.000 & \text { Peak 2: } & 6.713 & 99.8 & 1.156 \\ \text { Intercept: } 0.812 & \text { Peak 3: } & 0.000 & 0.0 & 0.000\end{array}$

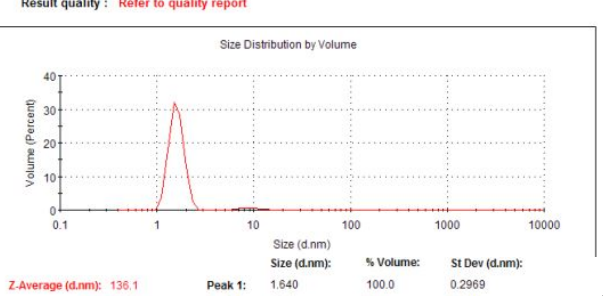

(c)

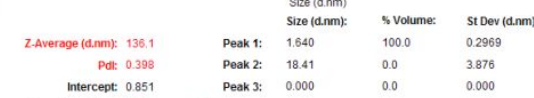

(d)

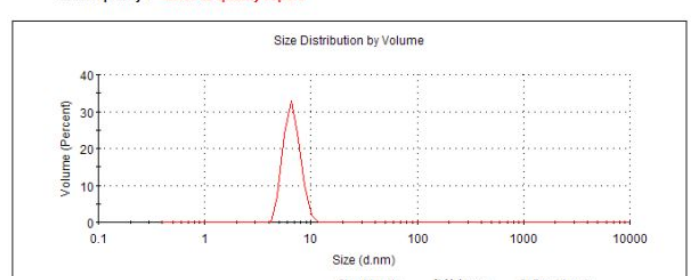
Result quality : Refer to quality repor
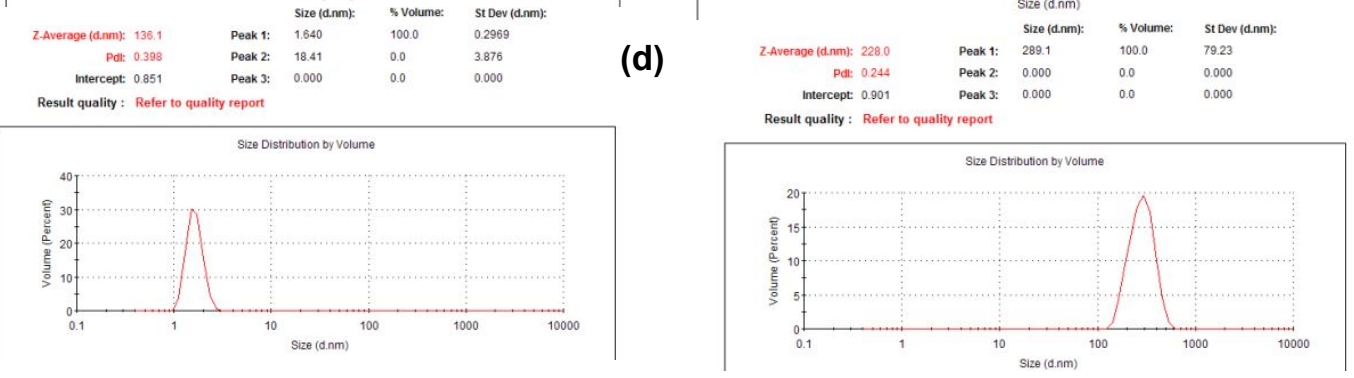

(e)
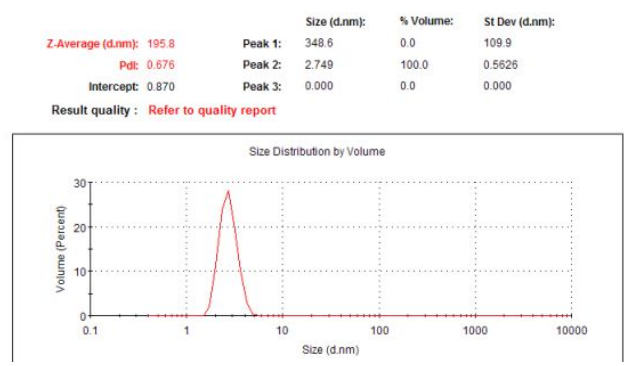

Figure S8. Particle size distributions of IQ-Six in different water content $(0 \% / \mathrm{a}, 30 \% / \mathrm{b}$, $60 \% / \mathrm{c}, 90 \% / \mathrm{d}$, and $99 \% / \mathrm{e}$ ). 

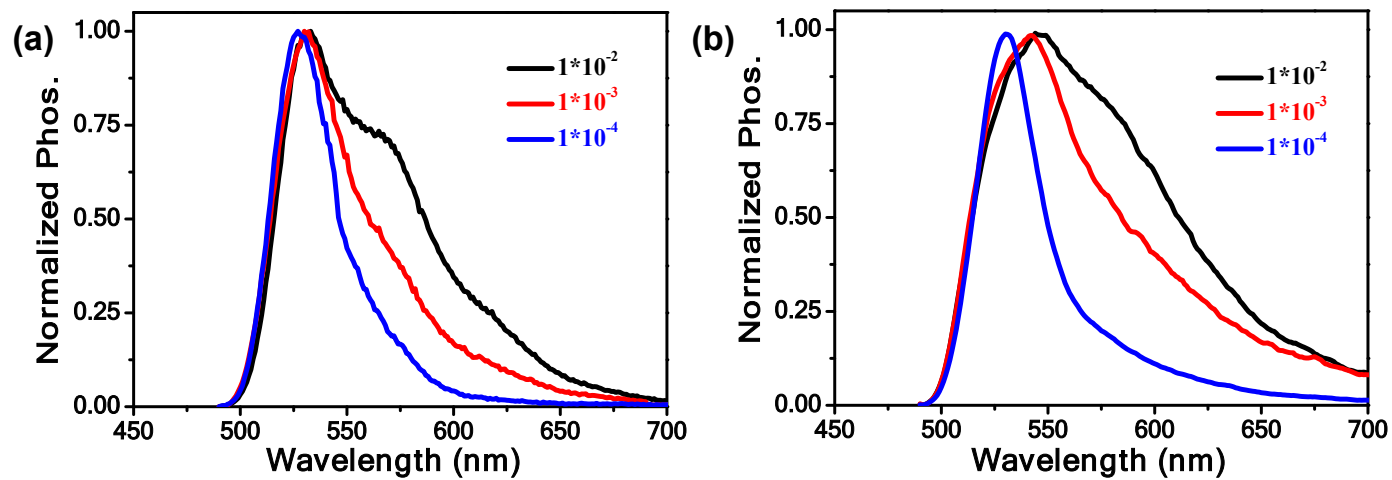

Figure S9. Phosphorescence spectra (77 K) of IQ-Five (a) and IQ-Seven (b) in THF solvent with different concentrations.
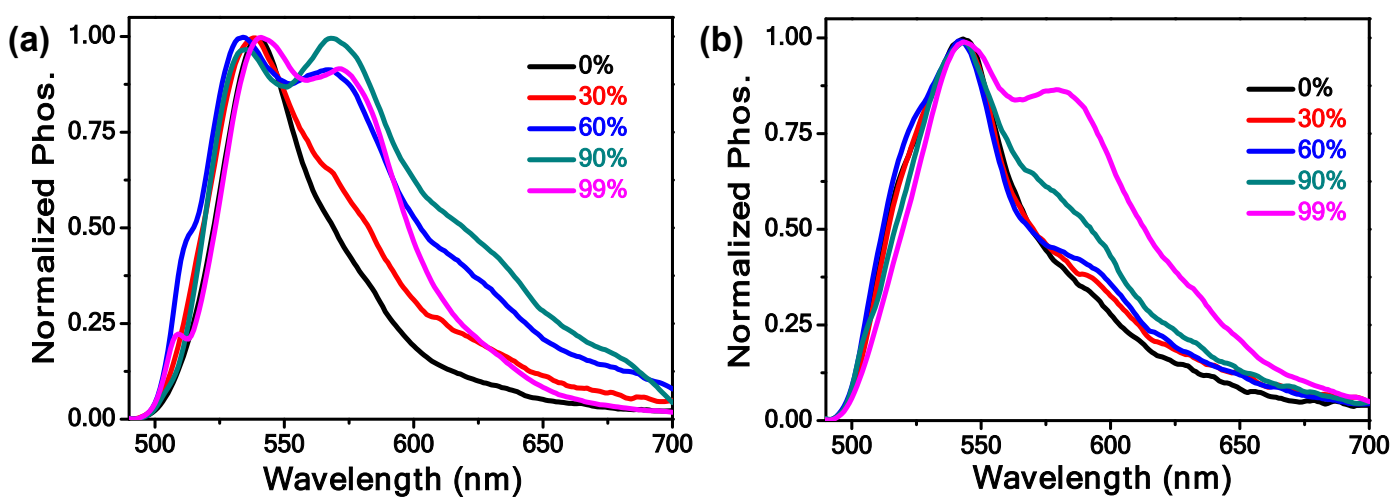

Figure S10. Phosphorescence spectra (77 K) of IQ-Five (a) and IQ-Seven (b) in $\mathrm{THF} / \mathrm{H}_{2} \mathrm{O}$ mixed solvent with different water contents.

Table S3. Average particle size and polydispersity index (PDI) of IQ-Five and IQSeven in THF-water mixtures with different fw.

\begin{tabular}{lccc}
\hline Compound & $f_{\mathrm{w}}$ & Average particle size $(\mathrm{nm})$ & PDI \\
\hline IQ-Five & $0 \%$ & 10.5 & 0.393 \\
& $30 \%$ & 106.3 & 1.000 \\
& $60 \%$ & 195.8 & 0.676 \\
IQ-Seven & $90 \%$ & 156.9 & 0.465 \\
& $99 \%$ & 136.1 & 0.398 \\
& $0 \%$ & 12.2 & 0.451 \\
& $30 \%$ & 75.3 & 1.000 \\
& $60 \%$ & 244.6 & 0.693 \\
& $90 \%$ & 221.2 & 0.491 \\
& $99 \%$ & 354.0 & 0.457 \\
\hline
\end{tabular}


(a)

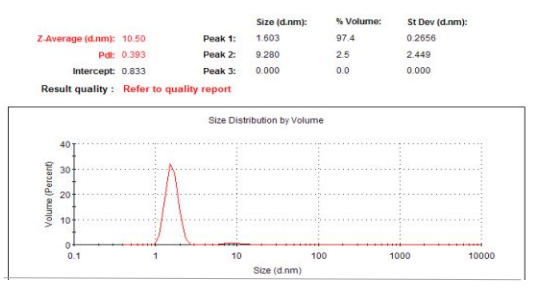

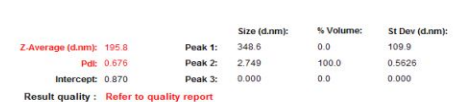

(c)

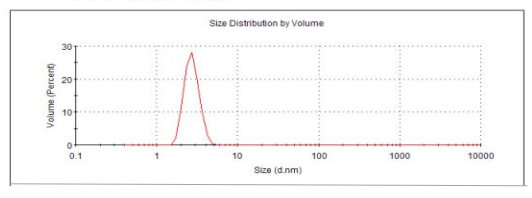

(d)

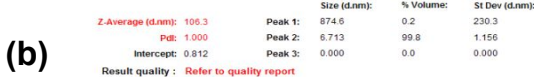

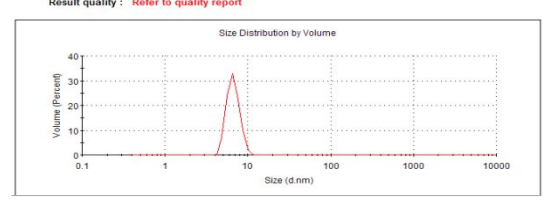

(e)

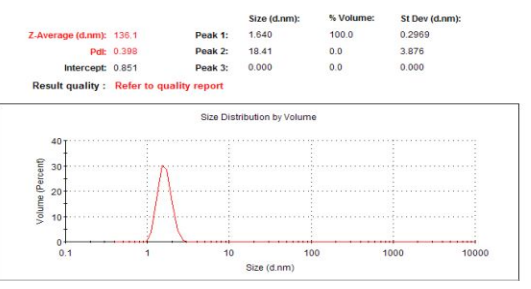

Figure S11. Particle size distributions of the IQ-Five in different water content $(0 \% / \mathrm{a}$, $30 \% / \mathrm{b}, 60 \% / \mathrm{c}, 90 \% / \mathrm{d}$, and $99 \% / \mathrm{e})$.

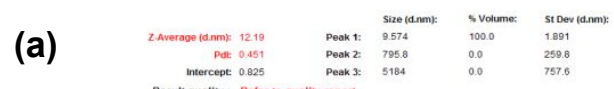

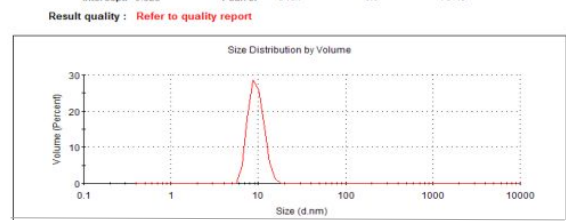

(c)

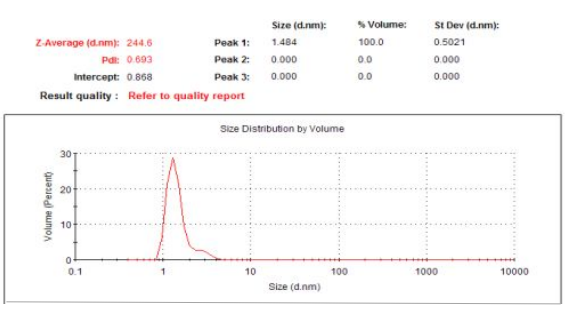

(b)

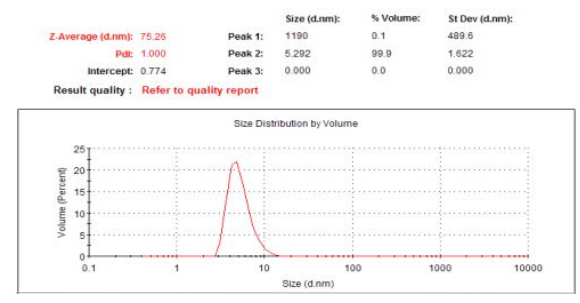

(d)
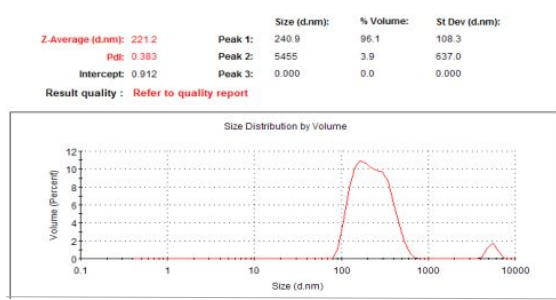

(e)

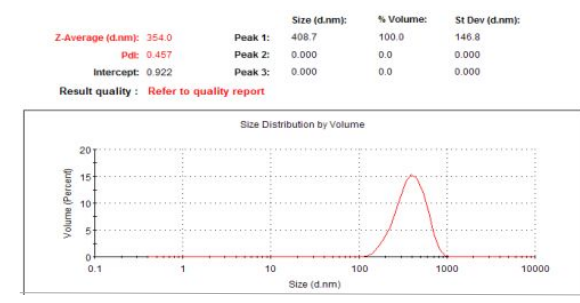

Figure S12. Particle size distributions of the IQ-Seven in different water contents (0\%/a, 30\%/b, 60\%/c, 90\%/d, and 99\%/e). 
(a)

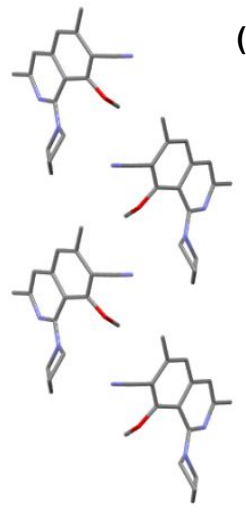

(b)

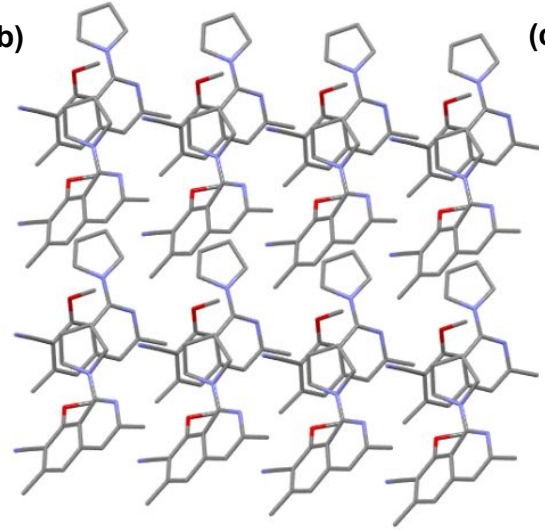

(c)

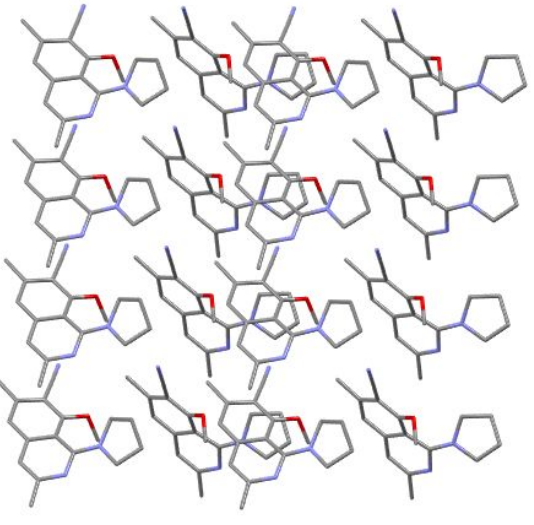

Figure S13. Packing patterns of IQ-Five along the a-axis (a), b-axis (b), and c-axis (c).

(a)

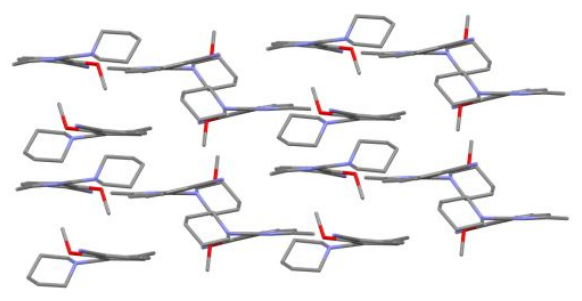

(b)

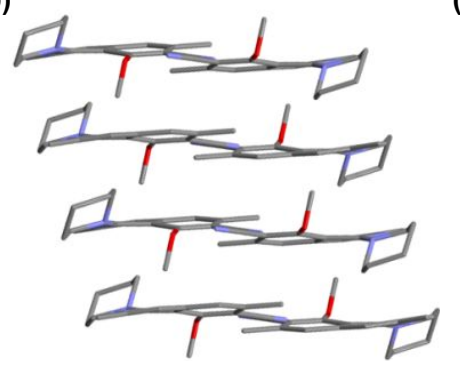

(c)

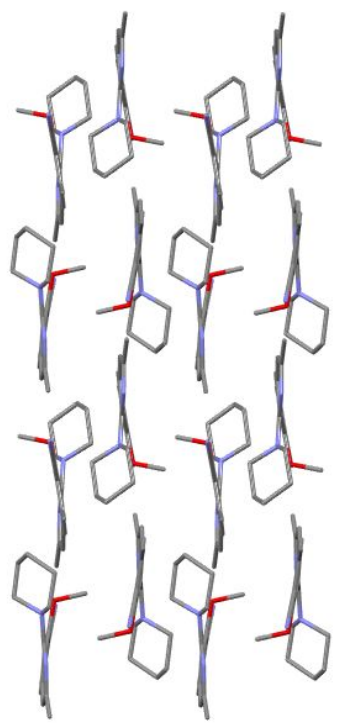

Figure S14. Packing patterns of IQ-Six along the a-axis (a), b-axis (b), and c-axis (c).

(a)

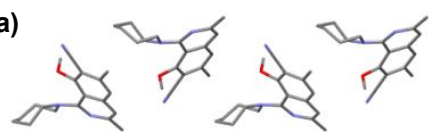
हो से हो से से कर से कर (b)

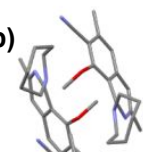

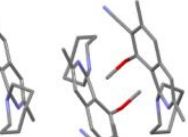<smiles>C1C2CC1C2</smiles>

(c)

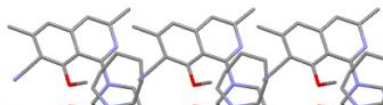

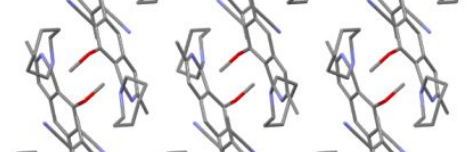
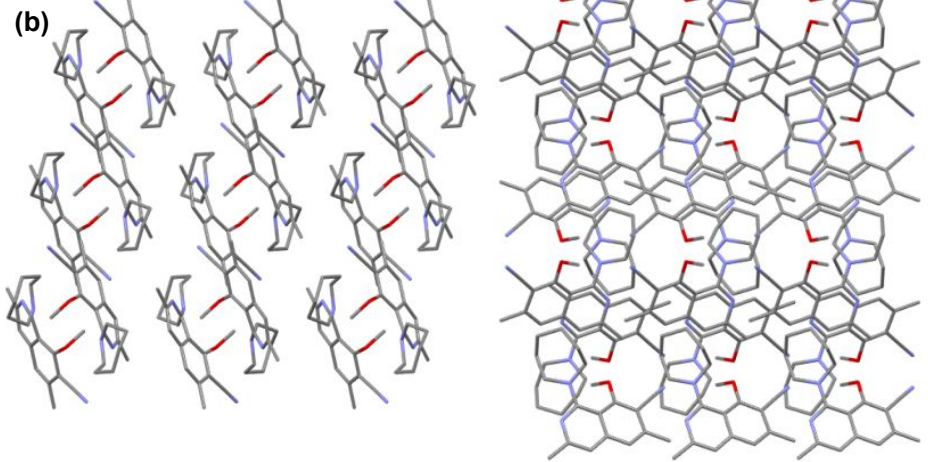

Figure S15. Packing patterns of IQ-Seven along the a-axis (a), b-axis (b), and c-axis (c). 


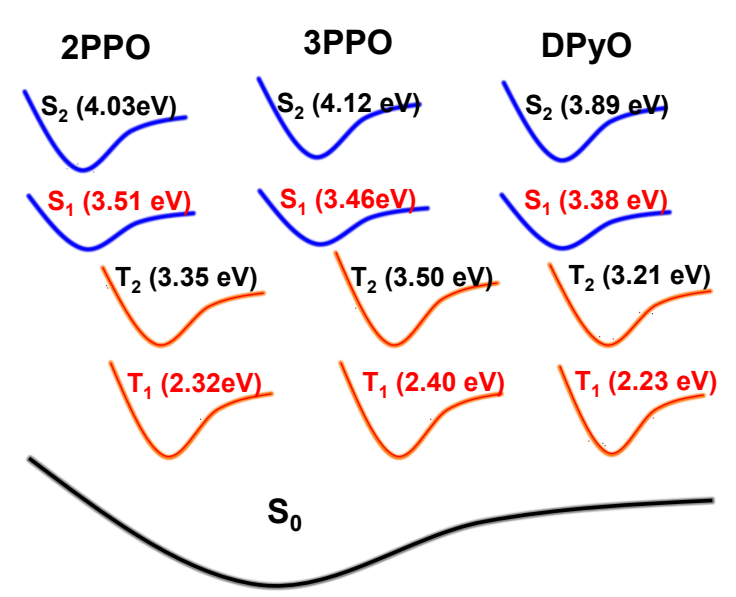

Figure S16. Energy levels of the hosts based on the theoretical calculation.

Table S4. Data of three guest single crystals.

\begin{tabular}{|c|c|c|c|}
\hline & IQ-Five & IQ-Six & IQ-Seven \\
\hline CCDC & 2086855 & 2032073 & 2086857 \\
\hline Empirical formula & $\mathrm{C}_{17} \mathrm{H}_{19} \mathrm{~N} 3 \mathrm{O}$ & $\mathrm{C}_{18} \mathrm{H}_{21} \mathrm{~N}_{3} \mathrm{O}$ & $\mathrm{C}_{19} \mathrm{H}_{23} \mathrm{~N}_{3} \mathrm{O}$ \\
\hline Formula weight & 281.35 & 295.38 & 309.40 \\
\hline Temperature (K) & $293(2)$ & $293(2)$ & 293(2) \\
\hline Crystal system & Monoclinic & Monoclinic & Monoclinic \\
\hline Space group & $P 2(1) / \mathrm{c}$ & $C 2 / \mathrm{c}$ & $P 2(1) / \mathrm{c}$ \\
\hline $\mathrm{Z}$ & 4 & 8 & 4 \\
\hline $\mathrm{D}_{\text {calcd }}\left[\mathrm{Mg} / \mathrm{m}^{3}\right]$ & 1.229 & 1.245 & 1.196 \\
\hline$F(000)$ & 600.0 & 1264 & 664 \\
\hline$\theta$ range $\left[{ }^{\circ}\right]$ & $3.242-25.997$ & $2.424-25.497$ & $2.375-26.000$ \\
\hline$R_{1}[\mathrm{I}>2 \sigma(\mathrm{I})]$ & 0.0498 & 0.0645 & 0.0537 \\
\hline$w R_{2}[\mathrm{I}>2 \sigma(\mathrm{I})]$ & 0.1261 & 0.1700 & 0.1149 \\
\hline$a[\AA]$ & $6.0201(2)$ & $31.204(7)$ & $7.2755(5)$ \\
\hline$b[\AA]$ & $22.9725(8)$ & $14.24(3)$ & $13.7706(9)$ \\
\hline$c[\AA]$ & $11.0281(4)$ & 7.0912(16) & $17.4597(13)$ \\
\hline$\alpha[\mathrm{deg}]$ & 90 & 90 & 90 \\
\hline$\beta[\operatorname{deg}]$ & $94.499(2)$ & $90.053(8)$ & $100.778(2)$ \\
\hline$\gamma[\mathrm{deg}]$ & 90 & 90 & 90 \\
\hline$V\left[\AA^{3}\right]$ & $1520.45(9)$ & $3152.3(12)$ & $1718.4(2)$ \\
\hline GOF & 1.052 & 1.057 & 1.032 \\
\hline$R$ (int) & 0.0233 & 0.0948 & 0.0538 \\
\hline No. of reflens collect & 7440 & 21336 & 12309 \\
\hline $\begin{array}{l}\text { No. of unique } \\
\text { reflcns }\end{array}$ & 2970 & 2936 & 3362 \\
\hline$R_{1}$ (all data) & 0.0660 & 0.0781 & 0.0960 \\
\hline$w R_{2}$ (all data) & 0.1396 & 0.1867 & 0.1403 \\
\hline
\end{tabular}


Table S5. Data of three host single crystals.

\begin{tabular}{|c|c|c|c|}
\hline & 2PPO & 3PPO & DPyO \\
\hline $\mathrm{CCDC}$ & 2086860 & 2086862 & 2086863 \\
\hline Empirical formula & $\mathrm{C}_{12} \mathrm{H}_{9} \mathrm{NO}$ & $\mathrm{C}_{12} \mathrm{H}_{9} \mathrm{NO}$ & $\mathrm{C}_{11} \mathrm{H}_{8} \mathrm{~N}_{2} \mathrm{O}$ \\
\hline Formula weight & 183.20 & 183.20 & 184.19 \\
\hline Temperature (K) & 293(2) & $293(2)$ & 293(2) \\
\hline Crystal system & Orthorhombic & Orthorhombic & Monoclinic \\
\hline Space group & $P 212121$ & $P$ n a 21 & $P 21 / \mathrm{n}$ \\
\hline $\mathrm{Z}$ & 4 & 4 & 4 \\
\hline $\mathrm{D}_{\text {calcd }}\left[\mathrm{Mg} / \mathrm{m}^{3}\right]$ & 1.280 & 1.276 & 1.311 \\
\hline$F(000)$ & 384 & 384 & 384 \\
\hline$\theta$ range $\left[{ }^{\circ}\right]$ & $3.071-26.000$ & $2.856-25.492$ & $2.878-25.996$ \\
\hline$R_{1}[\mathrm{I}>2 \sigma(\mathrm{I})]$ & 0.0342 & 0.0660 & 0.0594 \\
\hline$w R_{2}[\mathrm{I}>2 \sigma(\mathrm{I})]$ & 0.0799 & 0.1737 & 0.1542 \\
\hline$a[\AA]$ & $8.055(4)$ & $20.556(3)$ & $9.3063(17)$ \\
\hline$b[\AA]$ & $10.096(6)$ & $7.6031(12)$ & $10.2363(17)$ \\
\hline$c[\AA]$ & $11.687(5)$ & $6.1035(9)$ & $9.865(2)$ \\
\hline$\alpha[\mathrm{deg}]$ & 90 & 90 & 90 \\
\hline$\beta[\mathrm{deg}]$ & 90 & 90 & $96.832(8)$ \\
\hline$\gamma[\mathrm{deg}]$ & 90 & 90 & 90 \\
\hline$V\left[\AA^{3}\right]$ & $950.5(9)$ & $953.9(2)$ & $933.1(3)$ \\
\hline GOF & 1.035 & 1.049 & 1.041 \\
\hline$R$ (int) & 0.0326 & 0.0534 & 0.0527 \\
\hline No. of reflens collect & 7946 & 4035 & 4415 \\
\hline No. of unique reflens & 1845 & 1748 & 1810 \\
\hline$R_{1}($ all data $)$ & 0.0404 & 0.0855 & 0.0787 \\
\hline$w R_{2}$ (all data) & 0.0845 & 0.1966 & 0.1748 \\
\hline
\end{tabular}

Table S6. Phosphorescence quantum yields of the doped materials.

\begin{tabular}{lcc}
\hline \multicolumn{1}{c}{ Sample } & $\begin{array}{c}\text { Original } \\
(\%)\end{array}$ & $\begin{array}{c}\text { Ground } \\
(\%)\end{array}$ \\
\hline IQ-Five/2PPO & 8.4 & 6.1 \\
IQ-Six/2PPO & 15.2 & 12.4 \\
IQ-Seven/2PPO & 7.1 & 5.6 \\
IQ-Five/3PPO & 3.5 & 3.7 \\
IQ-Six/3PPO & 7.3 & 6.9 \\
IQ-Seven /3PPO & 3.1 & 3.3 \\
\hline
\end{tabular}

Note: Guest: Host $=1: 1000$ (Molar ratio). Excitation wavelength: $360 \mathrm{~nm}$. 


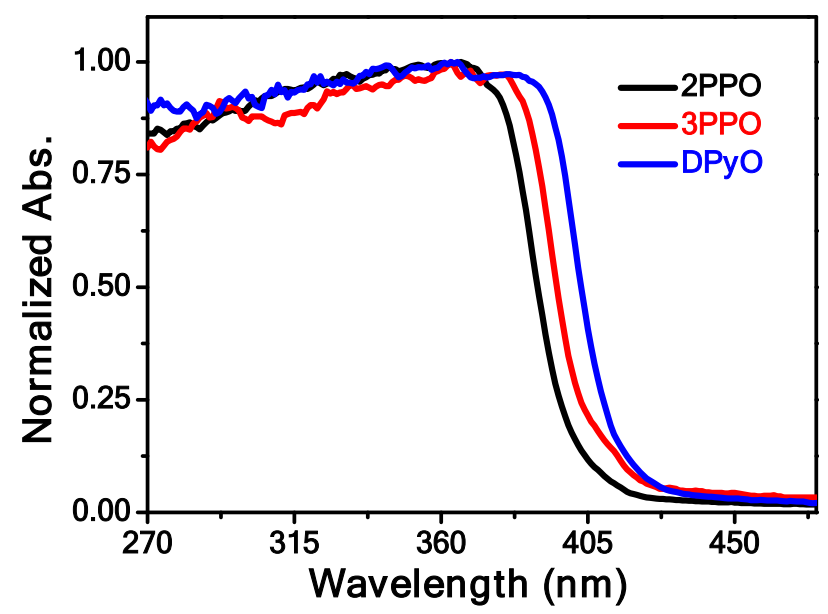

Figure S17. Absorption spectra of three hosts in solid state.
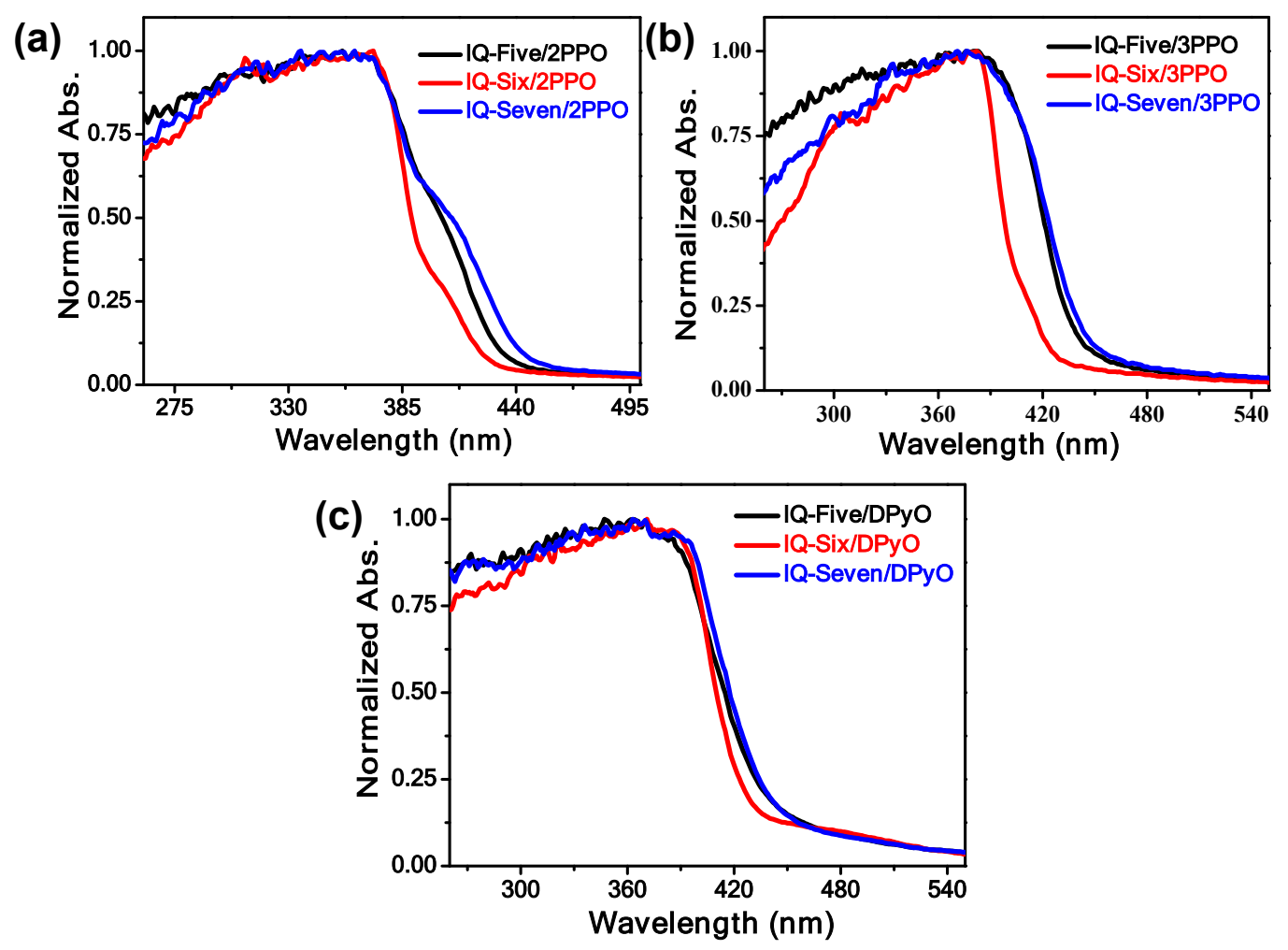

Figure S18. Absorption spectra of three guest-host systems. 


\section{NMR Spectra}

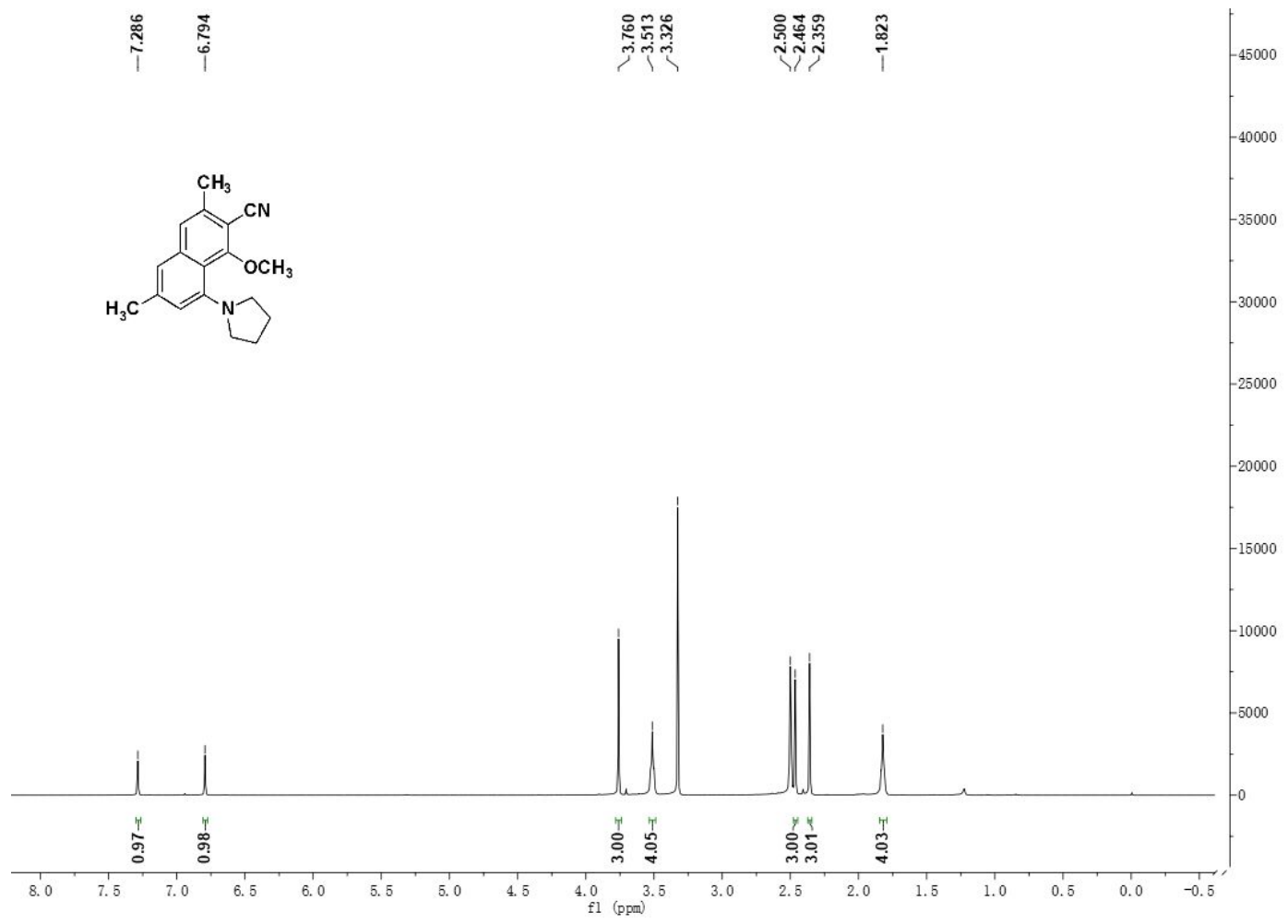

Figure S19. ${ }^{1} \mathrm{H}$ NMR of IQ-Five (DMSO- $\left.d_{6}, 500 \mathrm{MHz}\right)$.

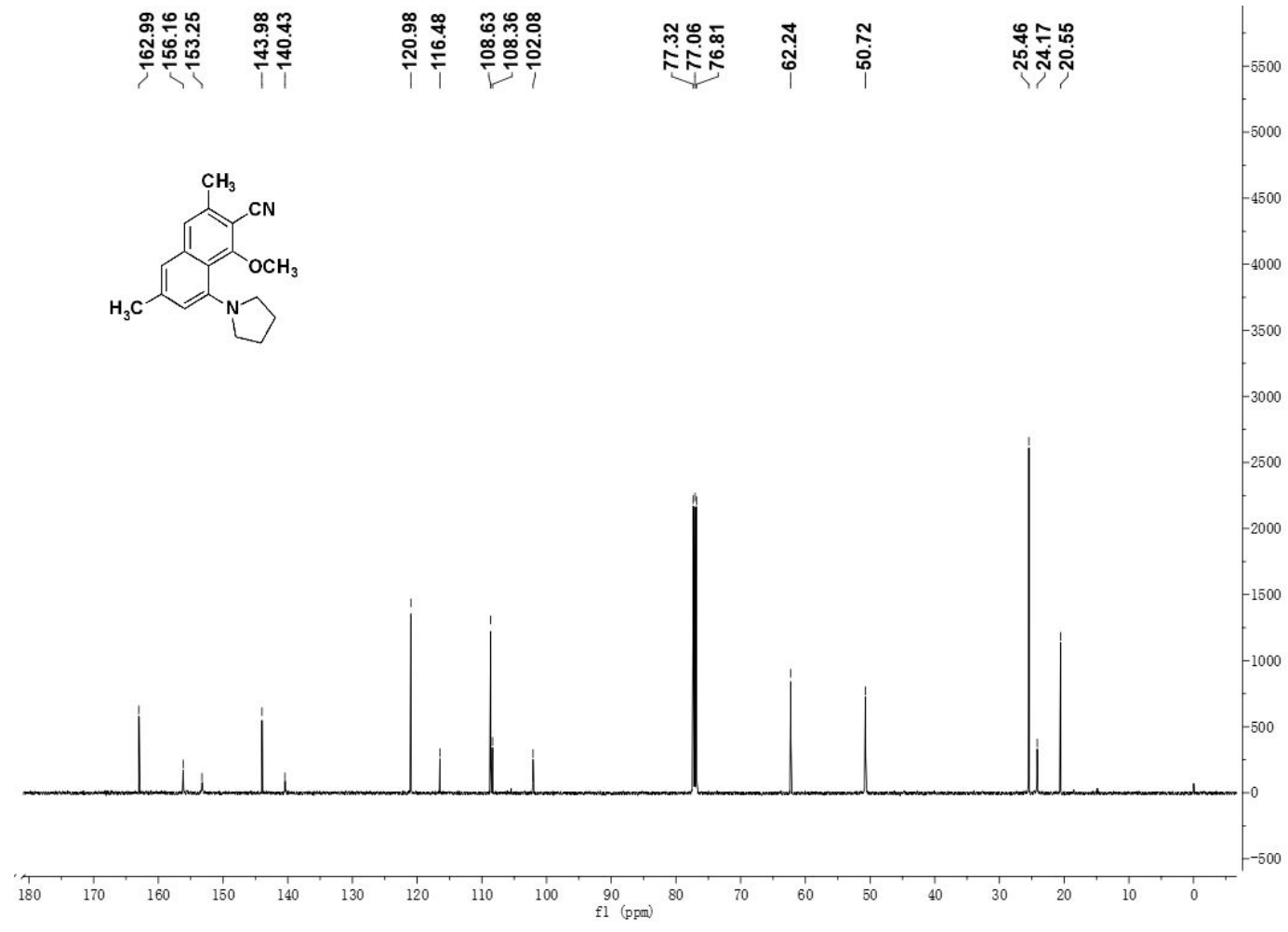

Figure S20. ${ }^{13} \mathrm{C} \mathrm{NMR}$ of IQ-Five $\left(\mathrm{CDCl}_{3}, 125 \mathrm{MHz}\right)$. 


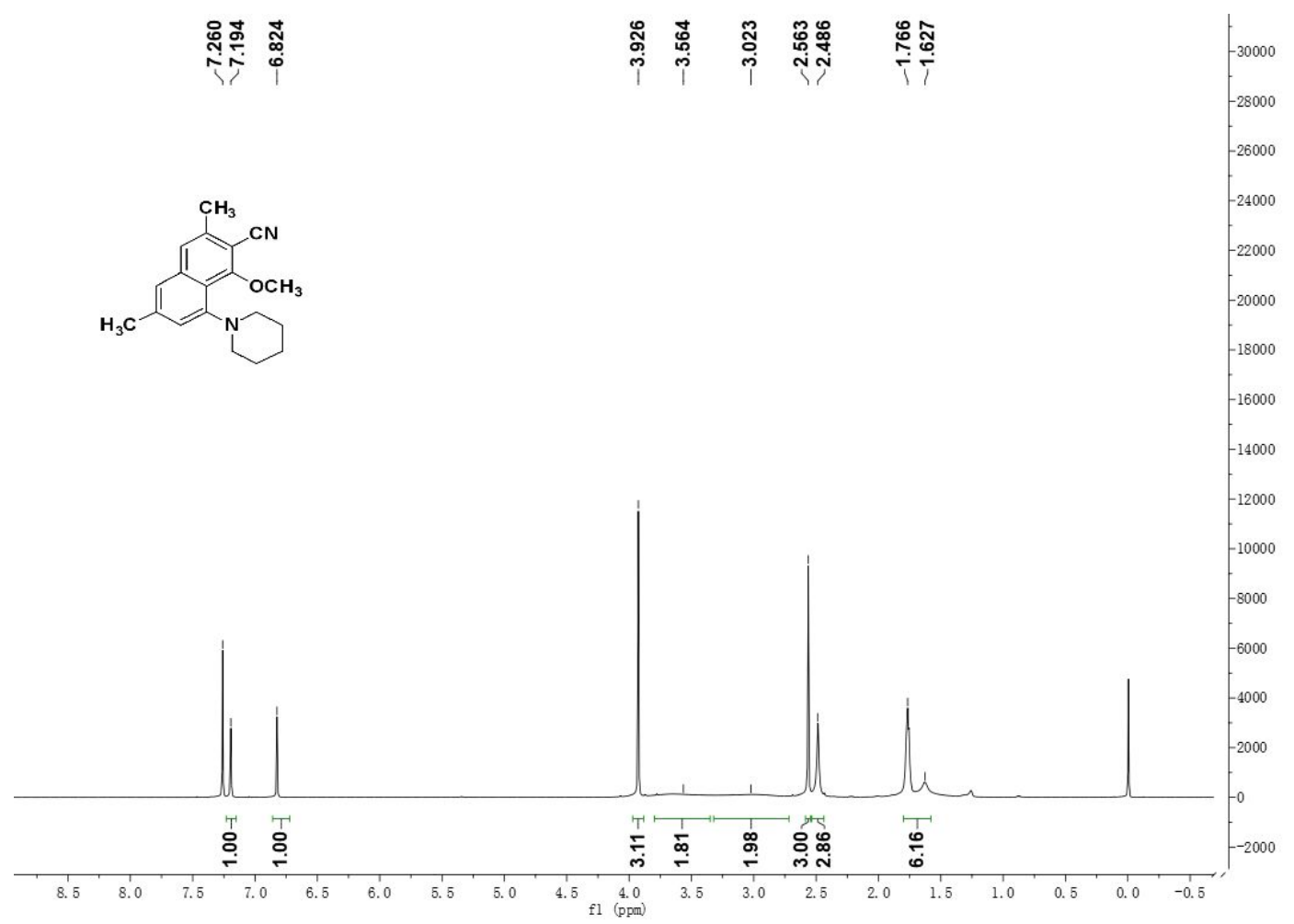

Figure S21. ${ }^{1} \mathrm{H}$ NMR of IQ-Six $\left(\mathrm{CDCl}_{3}, 500 \mathrm{MHz}\right)$.

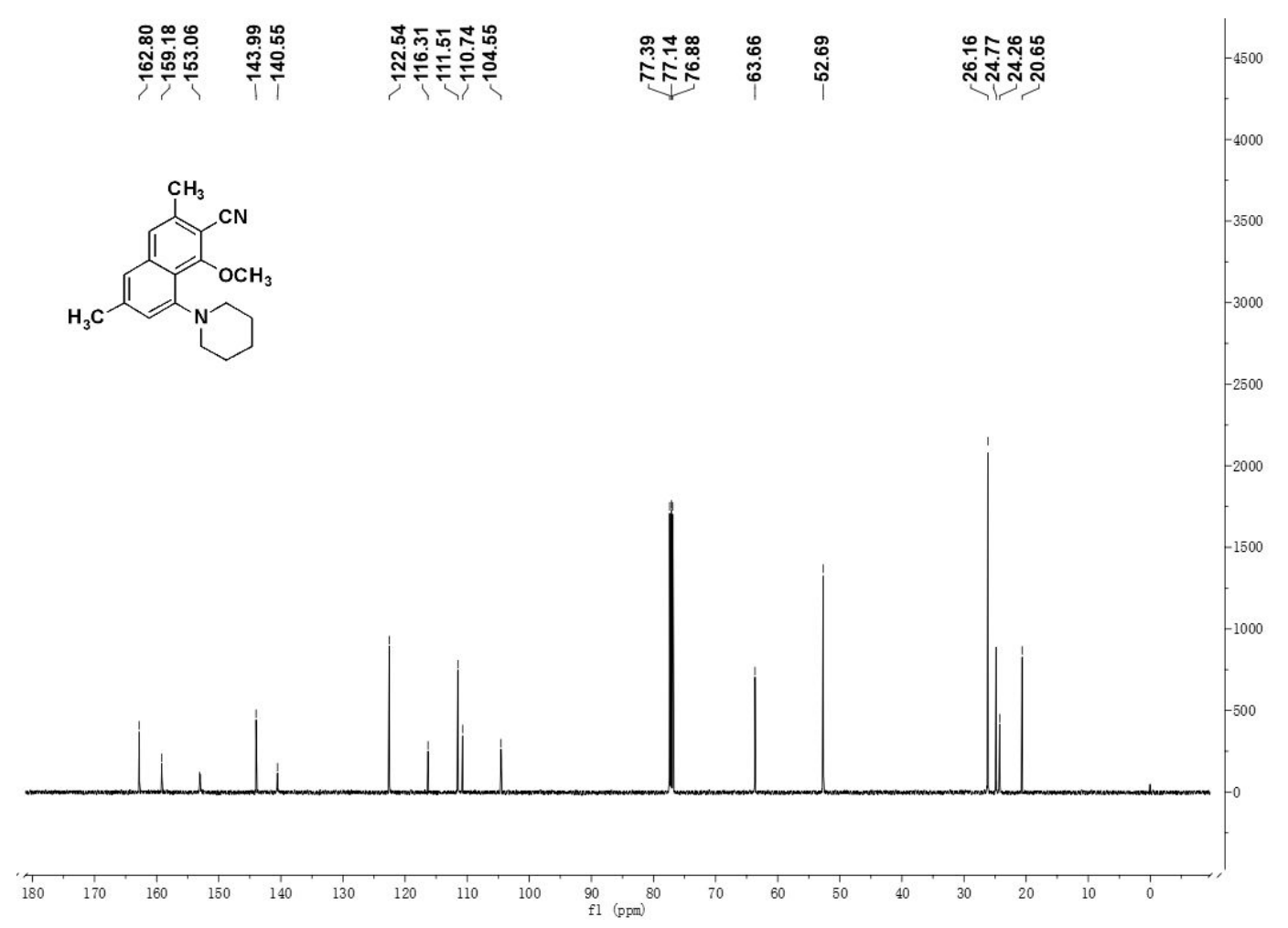

Figure S22. ${ }^{13} \mathrm{C}$ NMR of IQ-Six $\left(\mathrm{CDCl}_{3}, 125 \mathrm{MHz}\right)$. 


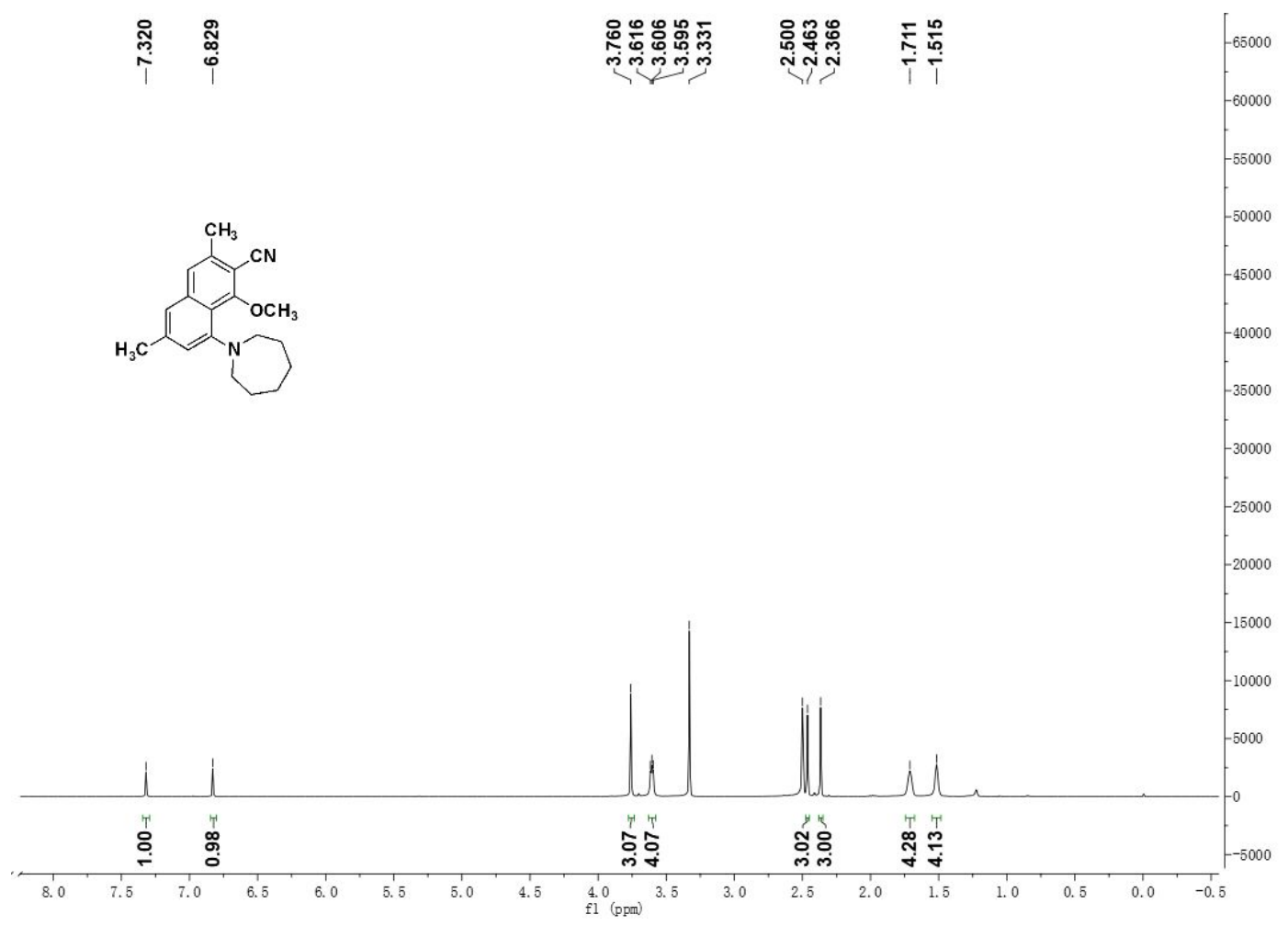

Figure S23. ${ }^{1} \mathrm{H}$ NMR of IQ-Seven (DMSO- $d_{6}, 500 \mathrm{MHz}$ ).

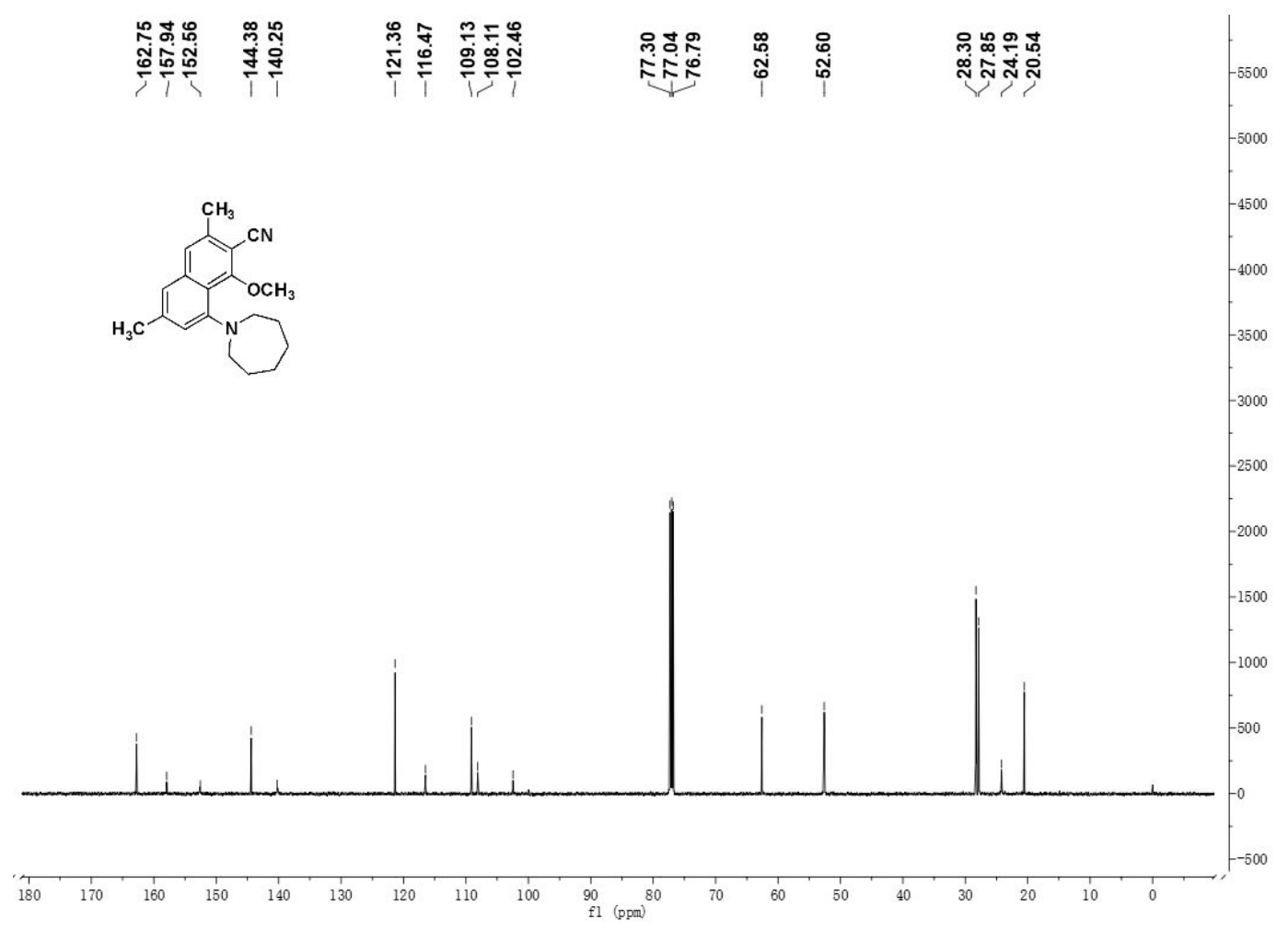

Figure S24. ${ }^{13} \mathrm{C}$ NMR of IQ-Seven $\left(\mathrm{CDCl}_{3}, 125 \mathrm{MHz}\right)$. 